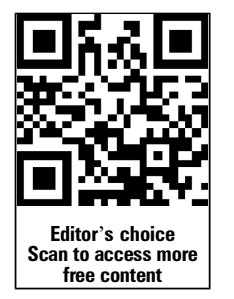

\title{
Low levels of 17- $\beta$-oestradiol, oestrone and testosterone correlate with severe evaporative dysfunctional tear syndrome in postmenopausal women: a case-control study
}

\author{
Caterina Gagliano, ${ }^{1,2}$ Salvatore Caruso, ${ }^{3}$ Giuseppe Napolitano, ${ }^{2}$ \\ Giulia Malaguarnera, ${ }^{4}$ Maria Vittoria Cicinelli, ${ }^{2}$ Roberta Amato, ${ }^{1,2}$ Michele Reibaldi, ${ }_{1}$ \\ Giuseppe Incarbone, ${ }^{5}$ Claudio Bucolo, ${ }^{4}$ Filippo Drago, ${ }^{4}$ Teresio Avitabile ${ }^{1}$
}

${ }^{1}$ University of Catania, Eye Clinic, Catania, Italy

${ }^{2}$ Neurovisual Science Technology (NEST), Catania, Italy

${ }^{3}$ Research Group for Sexology, Department of Medical and Surgical Specialties, University of Catania, Catania, Italy ${ }^{4}$ Department of Clinical and Molecular Biomedicine, Section of Pharmacology and Biochemistry, University of Catania, Catania, Italy ${ }^{5}$ Eidgenössische Technische Hochschule (ETH), Zürich, Switzerland

\section{Correspondence to}

Dr Caterina Gagliano, Neurovisual Science Technology (NEST), Via Tomaselli, Catania 43-95124, Italy; caterinagagliano@nestweb.it

Received 20 November 2012 Revised 1 October 2013 Accepted 3 November 2013 Published Online First 3 January 2014
CrossMark

\begin{tabular}{l}
\hline To cite: Gagliano C, \\
Caruso S, Napolitano G, \\
et al. Br J Ophthalmol \\
2014;98:371-376. \\
\hline
\end{tabular}

\begin{abstract}
Aims To evaluate the role of $17-\beta$-oestradiol, oestrone and total testosterone (TT) deficiency in the pathogenesis of severe evaporative dry eye syndrome (DES), investigating the relationship between tear osmolarity, tear film break-up time (TF-BUT), Schirmer test and serum sex hormones in postmenopausal women. Methods 44 postmenopausal women were recruited for a case-control study: 22 women with severe evaporative DES (Group A) and 22 without DES (Group B). The tests performed included laboratory blood analysis: fasting plasma profile (17- $\beta$-oestradiol, oestrone and TT), glucose level and lipid profile. Detailed eye examinations, including corneal and conjunctival staining, tear osmolarity measurement, tear volume and TF-BUT, were performed. The Ocular Surface Disease Index Questionnaire was also administered.

Results Values of Schirmer test and TF-BUT in Group A were significantly lower in comparison with Group B $(p<0.001)$. Serum levels of 17- $\beta$-oestradiol, oestrone and TT were significantly lower in Group A compared with Group $B(p<0.05)$. In women with severe evaporative DES, the levels of 17- $\beta$-oestradiol, oestrone and $\mathrm{TT}$ were inversely correlated with the tear film osmolarity ( $r=-0.7,-0.88,-0.81$, respectively).

Conclusions In postmenopausal women with severe evaporative DES, sex hormone levels are lower than control and that tear osmolarity is negatively correlated with sex hormone levels.
\end{abstract}

\section{INTRODUCTION}

Dry eye syndrome (DES) is the most frequent ocular complaint in elderly. It is characterised by eye irritation symptoms, blurred and fluctuating vision, tear film instability, increased tear osmolarity and ocular surface epithelial disease. ${ }^{1}$

DES may be caused by any disorder that decreases tear production (aqueous tear deficiency) or increases tear evaporation as in case of tear lipid deficiency. $^{2}$

The meibomian glands (MGs) secrete the lipid layer in human tears and lack of lipid component increases tear evaporation fourfold. ${ }^{3}$

Notably, DES affects women much more frequently than men. Women are more likely to experience DES during certain periods of significant hormonal alteration, for example, during pregnancy, lactation, oral contraceptive use and after menopause, suggesting that DES may be related to sex hormones. ${ }^{4-6}$

Receptors for androgens, oestrogens, progesterone and prolactin have been identified in several ocular tissues, including the lacrimal gland and MGs. ${ }^{7-9}$ Experimental and human studies have demonstrated that androgen levels are essential for normal lacrimal gland function and structural organisation and that prolactin and oestrogens also play important roles in providing an adequate hormonal milieu for optimal lacrimal production. ${ }^{10-12}$ However, few data exist on the role of sex hormones on lacrimal composition and MGs. Androgen receptors characterise MGs, a type of sebaceous gland, so it is hypothesised that these glands are under hormonal control. ${ }^{9}$ This point is particularly noteworthy because there is a significantly increased prevalence of meibomian gland disease (MGD) in postmenopausal women. Androgens act on the acinar epithelial cells, which contain receptor messenger RNA and/or androgen receptor protein. These cells respond to androgens by binding the androgen to a specific lipidproducing area on the cell, which then transcribes specified genes to increase the lipid layer distribution over the ocular surface..$^{9-12}$ On the contrary, an androgen deficiency or a topical anti-androgen treatment causes a significant decrease in the ocular lipid profile. ${ }^{13}$ The administration of sex hormones, such as the androgen precursor dehydroepiandrosterone, to the surface of the eye has been found to stimulate MG lipid production and release and prolong the eye's tear film break-up time (TF-BUT). ${ }^{10}$ The oestrogen produced after the menopause is primarily from the peripheral conversion of adrenal androgens to oestrone and occurs in the liver, kidney, brain, adrenal and peripheral adipose tissue. ${ }^{13}$ The absolute levels of oestrogen are influenced by weight, sex and age. Obese women have a higher concentration of free oestradiol because of decreased sex hormone-binding globulin and an increased rate of aromatisation. ${ }^{14}$ In postmenopause, androgens continue to be produced by the ovarian stromal and hilus cells in response to the increased levels of circulating lutein hormone. ${ }^{15}$ There is a $25 \%$ increase in testosterone secretion by the postmenopausal ovary; however, circulating levels of testosterone decrease to slightly less than those of premenopausal women. This 
reduction has been attributed to a significant fall in the conversion of androstenedione to testosterone after the menopause. ${ }^{11}$

The result is sex hormone deficiency, like that occurring in menopause that may alter not only aqueous lacrimal production but also MG function and lipid production leading to increased evaporation rate and occurrence of DES in its evaporative form. ${ }^{14-18}$

The aim of this study was to examine the relationship between tear osmolarity, tear volume, TF-BUT and sex hormones serum levels (17- $\beta$-oestradiol, oestrone and total testosterone (TT)) in postmenopausal women with severe evaporative DES.

\section{METHODS}

The study was conducted according to a protocol approved by local ethics committee 'Azienda Ospedaliera Universitaria Vittorio Emanuele Policlinico Catania' and conformed to the ethical guidelines of the 1975 Helsinki Declaration and the International Conference of Harmonization (ICH) guideline for Good Clinical Practice. Written informed consent was obtained from each woman before entering the study.

\section{Setting and sample}

The case-control study was performed at Neurovisual Science Technology (NEST) Laboratory of Clinical Ophthalmology, Catania, Italy, and at Ophthalmic Clinic, Department of Medical Surgical Specialties, University of Catania, School of Medicine, Catania, Italy. It was conducted during the period from November 2011 to April 2012 on 44 postmenopausal volunteer women aged 52-63 years (mean age 57 \pm 3.9 ), as defined as in amenorrhoea since at least 1 year, with serum follicle stimulating hormone (FSH) levels higher than $12 \mathrm{mIU} / \mathrm{mL}$ and oestradiol levels lower than $15 \mathrm{pg} / \mathrm{mL}$. Volunteers were recruited from a hospital clinic who were later subdivided into two groups: 22 women with severe evaporative DES (Group A) and 22 without DES (Group B). The sample size (at least 20 eyes for each group) was determined from the results of our preliminary data to detect, with an $\alpha$ of 0.05 and a $90 \%$ power (two-tailed), a $15 \%$ difference in each parameter. $\mathrm{p}$ Values lower than 0.05 were considered as statistically significant. We use independent samples $t$ test to compare the means of two samples (Group A and Group B) in relation to the variation in the data (expressed as the SD of the difference between the means).

Statistical analysis was performed using Systat V.13.0 (SSPS, Chicago, Illinois, USA).

At enrolment, physical and clinical examinations were performed and medical, surgical and medication histories were assessed to ensure study eligibility on the basis of inclusion and exclusion criteria. Women with a history of eye disease or previous surgery; use of hormonal, systemic or topical antiinflammatory and/or antibiotic therapies; use of an experimental drug 30 days or less prior to study entry and use of tobacco were excluded from the study.

The evaluations included the following examinations:

- Body weight: patients were weighed after urinating, wearing only underclothing (which was accurate to $\pm 0.1 \mathrm{~kg}$ ) for all measurements.

- Laboratory blood analysis: blood samples were drawn at 8:00 for determination of fasting plasma hormone profile (17- $\beta$-oestradiol, oestrone and TT), glucose level and lipid profile (serum triglycerides, total cholesterol and high-density lipoprotein (HDL)-cholesterol).

- Nutrient analysis: patients individually completed a questionnaire (Frequency of Food Consumption) composed of a total of 144 foods to establish the type of nutrients usually consumed.

- Detailed eye examinations, including corneal staining with fluorescein (evaluated using the National Eye Institute method, a standardised scale (0-3) for each of the five regions of the cornea: central, inferior, nasal, superior and temporal) and conjunctival staining with lissamine green (evaluated using the Oxford Schema (0-4) for three regions of the conjunctiva: central, nasal and temporal). During anterior segment slit-lamp examination, inspection of eyelid margins and conjunctiva was done carefully taking note of hyperaemia of the bulbar and palpebral conjunctiva, lid wiper epitheliopathy, lid parallel conjunctival folds and keratinisation of the MG ductal epithelium (ie, orifice metaplasia) and of the lids.

- Tear osmolarity: each patient was seat with head back and eyes upwards towards the ceiling, and $50 \mathrm{~nL}$ of tear sample was collected from the lower eyelid and the line of moisture along the inner eyelid margin, near the lateral cantus of both eyes, using Tearlab Osmolarity System Pen (TearLab Inc, San Diego, California, USA), holding a single-use Test Card. Then, the tear film osmolarity of each sample was measured by using the Tearlab Osmolarity System Reader (TearLab Inc). Normal values range between 289 and $308 \mathrm{mOsm} / \mathrm{L}$, in a DES patient range between 312 and $378 \mathrm{mOsm} / \mathrm{L}{ }^{19}$

- Schirmer test, performed for 5 min with anaesthesia with the patient's eyes closed and calculated in millimetres.

- Fluorescein TF-BUT, measured as the time from a patient's blink to the appearance of the first dry spot on the cornea and calculated in seconds.

- Ocular Surface Disease Index (OSDI) Questionnaire.

Patients were authorised to use artificial tears during the study, but were instructed not to change the type of drop and not to use it on the visit day.

Twenty-two volunteer women suffering from severe evaporative DES, due to MGD (stage 4 as reported in the classification of Geerling et $a l^{20}$ as defined by "a chronic, diffuse abnormality of the MGs, commonly characterised by terminal duct obstruction and/or qualitative/quantitative changes in the glandular secretion" and abnormal TF-BUT) constituted Group A.

Twenty-two women with aqueous deficiency and no evidence of MGD were considered as control group (Group B). For each woman, data from right eye were included in the study.

\section{RESULTS}

All 44 women were of normal and stable weight (mean weight $71.6 \mathrm{~kg}$, range $60.5-80.6$, mean body mass index $22.5 \mathrm{~kg} / \mathrm{m}^{2}$, range 20.0-25.0) and there was no significant difference between Group A and Group B in terms of patient age, years since menopause, weight, plasma lipid profile (triglycerides, total cholesterol and HDL-cholesterol), fasting plasma glucose and average daily intake of nutrients (table 1 ).

Table 2 shows the serum levels of 17- $\beta$-oestradiol, oestrone and TT of the two groups. Sex hormones were significantly lower in women affected by DES compared with control women $(\mathrm{p}<0.05)$.

Figure 1 shows the correlation between sex hormone levels and tear osmolarity. The levels of $17-\beta$-oestradiol, oestrone and TTwere inversely correlated with tear film osmolarity ( $\mathrm{r}$ values $=$ $-0.7,-0.88,-0.81$, respectively). A positive correlation has been demonstrated between Schirmer values ( $\mathrm{r}$ values $=0.69$, $0.83,0.74)$ (figure 2$)$ as well as TF-BUT $(r=0.76,0.87,0.75)$ (figure 3 ) and sex hormone levels. 
Table 1 Age, years since menopause, fasting data on weight, lipid profile and plasma glucose are summarised

\begin{tabular}{lcc}
\hline Parameters & $\begin{array}{l}\text { Group A (DES) } \\
(\mathbf{n}=\mathbf{2 2})\end{array}$ & $\begin{array}{l}\text { Group B (control) } \\
(\mathbf{n}=\mathbf{2 2})\end{array}$ \\
\hline Age (years) & $58.2 \pm 3.2$ & $57.6 \pm 2.6$ \\
Years since menopause (years) & $8.5 \pm 2.4$ & $7.8 \pm 1.9$ \\
Weight (kg) & $72.5 \pm 2.0$ & $72.4 \pm 2.0$ \\
Triglycerides (mmol/L) & $1.0 \pm 0.1$ & $0.8 \pm 0.1$ \\
Total cholesterol (mmol/L) & $4.9 \pm 0.2$ & $4.5 \pm 0.2$ \\
HDL-cholesterol (mmol/L) & $1.2 \pm 0.1$ & $1.1 \pm 0.1$ \\
Fasting plasma glucose (mmol/L) & $5.0 \pm 0.1$ & $4.8 \pm 0.1$ \\
\hline No significant differences ( $\mathbf{p}>0.05)$ were observed between the two groups. \\
DES, dry eye syndrome; HDL, high-density lipoprotein.
\end{tabular}

Table 2 Serum levels of 17- $\beta$-oestradiol, oestrone and TT of the two groups

\begin{tabular}{lll}
\hline & Group A (DES) $(\mathrm{n}=22)$ & Group B (control) $(\mathrm{n}=\mathbf{2 2})$ \\
\hline 17- $\beta$-oestradiol $(\mathrm{pg} / \mathrm{mL})$ & $12.5 \pm 1.9$ & $14.4 \pm 2.1$ \\
Oestrone $(\mathrm{pg} / \mathrm{mL})$ & $41.0 \pm 0.4$ & $46.8 \pm 0.16$ \\
Testosterone $(\mathrm{ng} / \mathrm{mL})$ & $31.9 \pm 0.4$ & $35.5 \pm 0.2$ \\
\hline
\end{tabular}

Sex hormones were significantly lower in women affected by DES compared with control women $(p<0.05)$

$\mathrm{DES}$, dry eye syndrome; TT, total testosterone.

Figure 4 shows the comparison between the tear film osmolarities in the two groups. The mean tear osmolarities in Groups $\mathrm{A}$ and $\mathrm{B}$ were $378.77 \pm 8.48$ and $309.18 \pm 13.47 \mathrm{mOsmol} / \mathrm{L}$, respectively. Tear osmolarity was significantly higher in Group A in comparison with controls $(p<0.001 ; t=20$ 494). There were statistically significant differences between the two groups in terms of Schirmer test value $(\mathrm{p}<0.001 ; \mathrm{t}=142$ 663) (figure 5) and TF-BUT value $(\mathrm{p}<0.001 ; \mathrm{t}=297419)$ (figure 6).

Group A also tended to have worse scores than Group B on self-reported symptoms as measured by OSDI $(43.3 \pm 16.42$ vs $28.5 \pm 18.34 ; \mathrm{p}<0.001)$. Differences in lissamine green, fluorescein staining and hyperaemia on the ocular surface (lid margin, MGs and conjunctiva) between the two groups were statistically significant $(\mathrm{p}<0.001)$.

\section{DISCUSSION}

Several data in literature suggest a significant influence of gender and sex hormones on the physiology of the lacrimal gland and that they are involved in the pathogenesis of DES. Interestingly, DES occurs mainly in women and its appearance is frequently related to marked variations in serum sex hormone levels like menopause. ${ }^{21}$

The results of this study showed that the serum levels of $17-\beta$-oestradiol, oestrone and TT were significantly lower in women affected by DES compared with control. Moreover, the levels of all sex hormones investigated were inversely correlated with tear osmolarity ( $\mathrm{rs}=-0.7,-0.88,-0.81$, respectively). Tear film hyperosmolarity, a recognised component for the definition of dry eye by Dry Eye WorkShop, ${ }^{1}$ recently was found to be the single best marker of disease severity and marker of disease progression. $^{22}$

This inverse correlation of sex hormone levels and tear film osmolarity adds further evidence on the role of sex hormone levels in the pathogenesis of DES. In particular, sex hormone deficiency may cause MGD. Meibum lipids promote the tear film stability and prevent tear evaporation, maintaining health and integrity of the ocular surface. ${ }^{23} 24$ The dysfunction of this gland leads to lipid insufficiency, tear film instability, hyperosmolarity and increasing evaporation of the tear film. ${ }^{25}$

MGD leads to lipid insufficiency, increasing evaporation, instability and hyperosmolarity of the tear film. Our findings are
Figure 1 Correlation between sex hormone levels and tear osmolarities. The levels of 17- $\beta$-oestradiol, oestrone and total testosterone were inversely correlated with tear film osmolarity $(r$ values $=-0.7 ;-0.88 ;-0.81$, respectively).

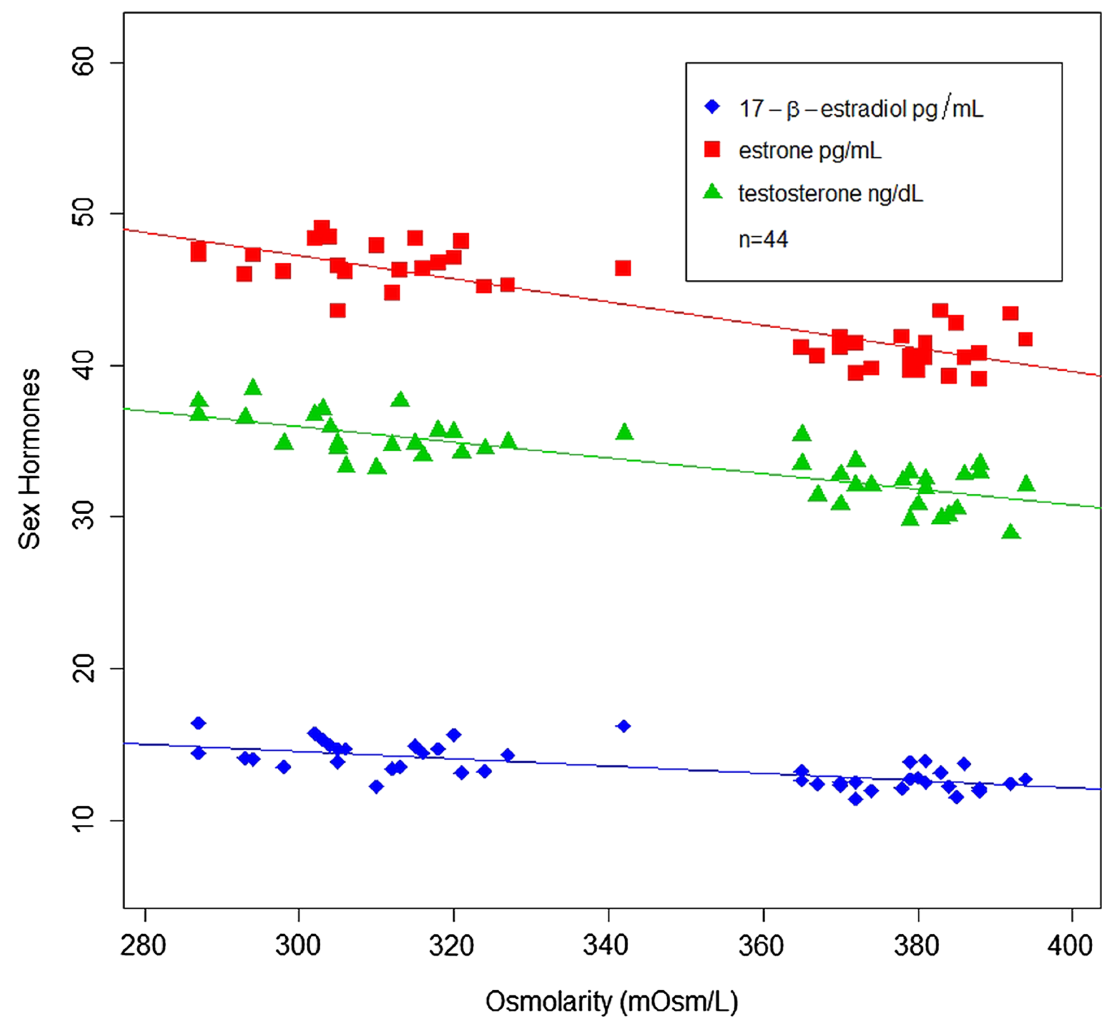


Figure 2 Direct correlation between plasma hormone levels

(17- $\beta$-oestradiol, oestrone and testosterone) and Schirmer test values $(r=0.69 ; 0.83 ; 0.74$, respectively).

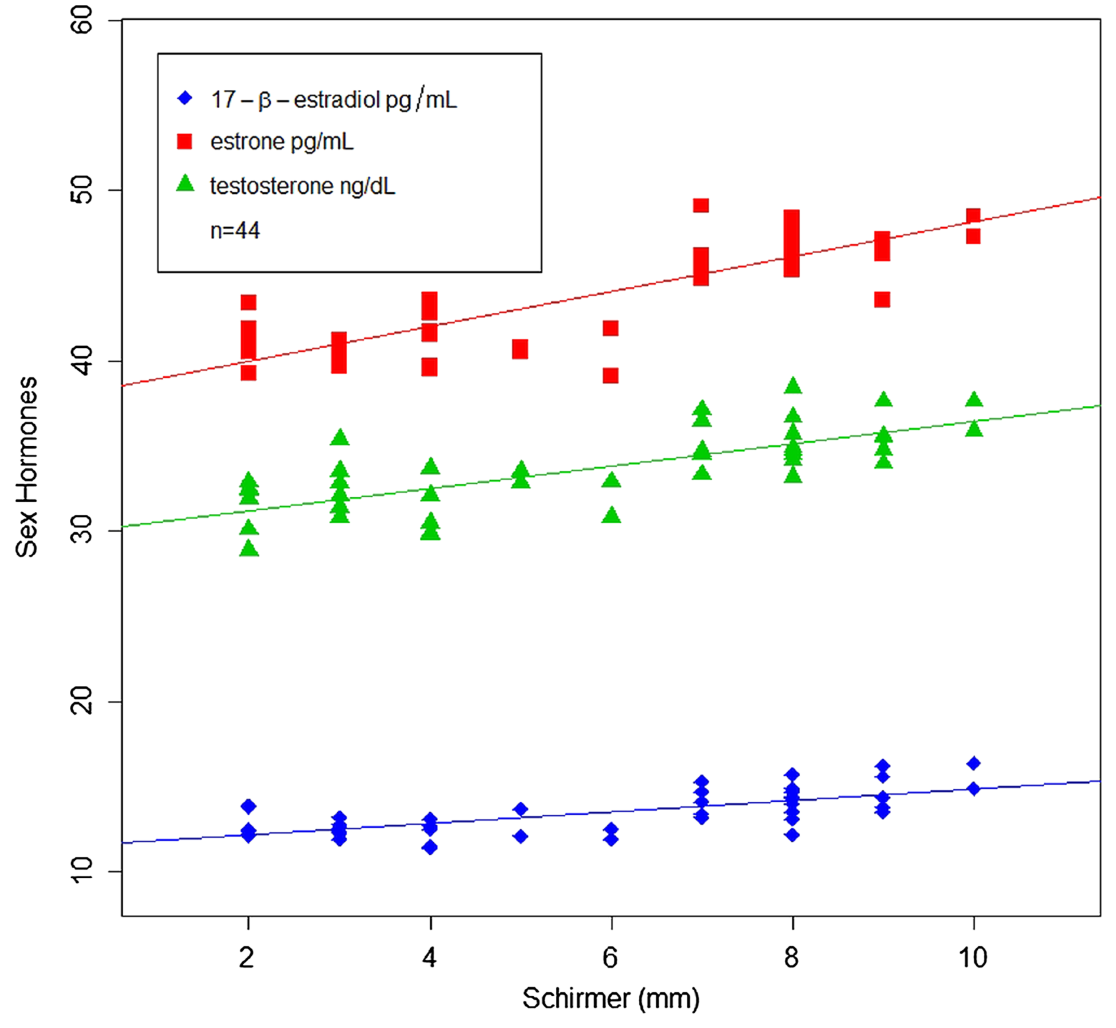

in accordance with previous evidence indicating that both testosterone and 17- $\beta$-Oestradiol influence and regulate MG integrity and functions, enhance the quality and/or quantity of lipids produced by MGs and promote the formation of the tear film's lipid layer in humans. ${ }^{26} 27$

The data from this study suggest that deficiency in sexual hormones may cause not only a reduction in tear production leading to aqueous-deficient dry eye, but also a dysfunction in MG function determining an evaporative dry eye. The mRNA for sex steroid receptors has been demonstrated in MGs in humans suggesting that these glands are target organs for androgens, oestrogens and/or progestins. ${ }^{7}{ }^{28}$ Sex steroid deficiency may alter MG function in different ways by altering secretion of the lipid components or also acting throughout an inflammatory
Figure 3 Direct correlation between plasma hormone levels (17- $\beta$-oestradiol, oestron, and testosterone) and tear film break-up time (TF-BUT) values $(r=0.76 ; 0.88$; 0.76 , respectively).

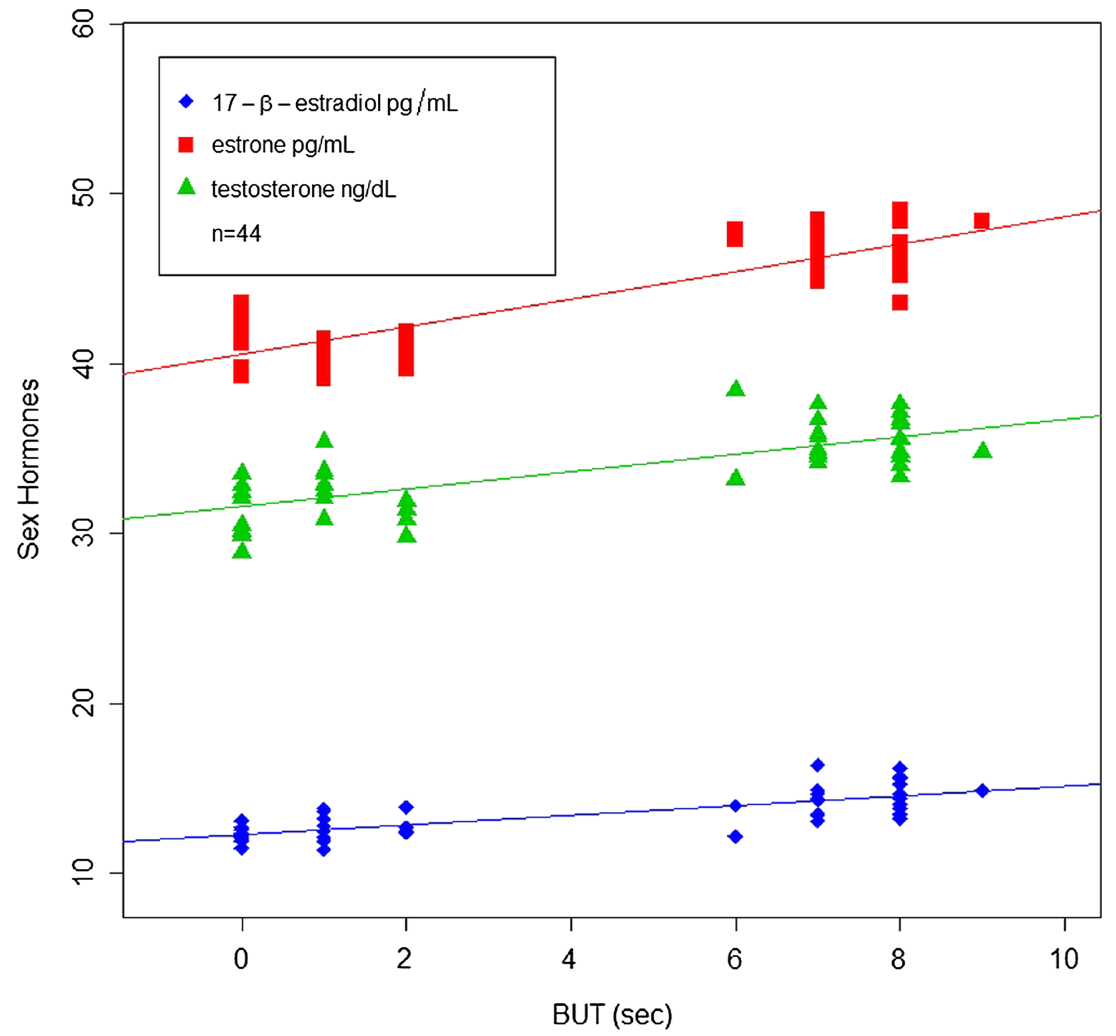




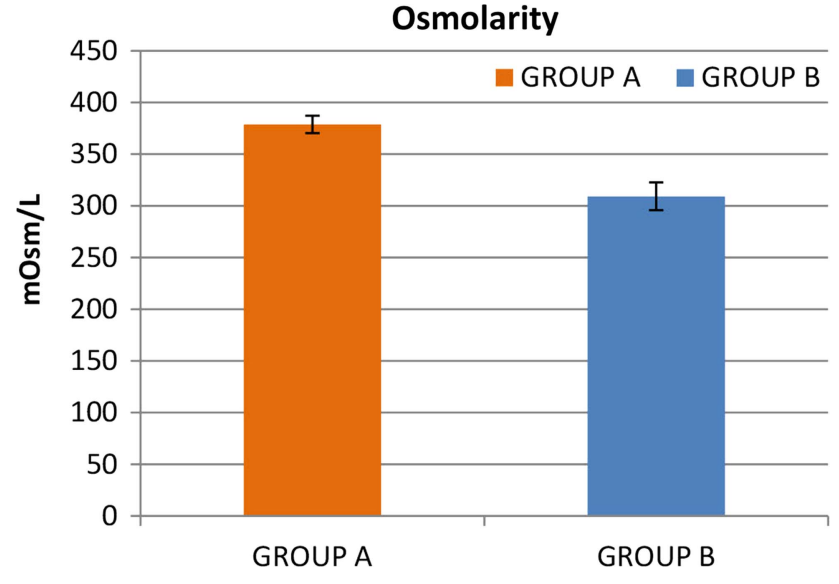

Figure 4 Tear osmolarity was significantly higher in Group $A$ in comparison with controls ( $p<0.001 ; t=20494)$. Error bars represent SD.

process. ${ }^{17} 2930$ In particular, Suzuki et $a l^{31}$ demonstrated that testosterone stimulates genes related to lipid metabolic pathways and suppresses genes regulating epithelial keratinisation. In women with complete androgen insensitivity syndrome due to androgen receptor dysfunction, an alteration in neutral and polar lipid patterns of human MG secretion along with a keratinisation of the MG ductal epithelium (ie, orifice metaplasia) and the lid has been observed. ${ }^{27}$ Notably, in these women there was a significant increase in signs and symptoms of dry eye. ${ }^{28}$ Data in literature show that serum sex hormone levels in postmenopausal women are influenced by age, years since menopause and weight and that oestradiol and androgen levels are negatively correlated with age. ${ }^{32}$ Notably, all these parameters were not significantly different in the two groups of women. However, it must be underlined that elevated androgen levels may also be responsible for MGD. In fact, a higher incidence of evaporative dry eye (EDE) has been observed in hyperandrogenic women affected by polycystic ovarian syndrome. ${ }^{28}$ This allows us to speculate that lacrimal function as well as other functions in the visual system is profoundly influenced by sex hormones and that a fine equilibrium between androgens and oestrogens is needed for a normal function. ${ }^{33} 34$

In conclusion, this study demonstrates that postmenopausal women are at risk for DES and that low levels of sex steroids, both androgens and oestrogens, are related to the occurrence of dry eye in its evaporative form. These findings allow for the hypothesis that hormone treatment may be useful to increase

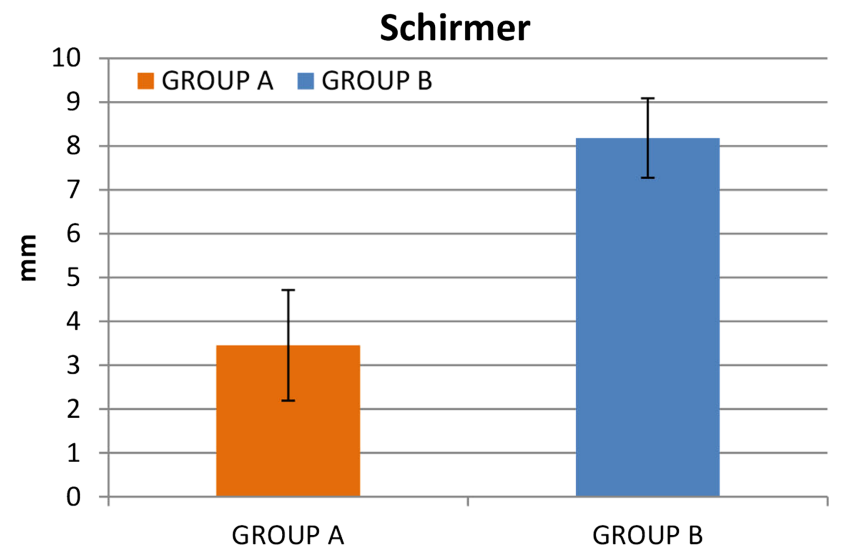

Figure 5 Statistically significant differences between the two groups for Schirmer test values $(p<0.001 ; t=142663)$. Error bars represent SD.
TF-BUT

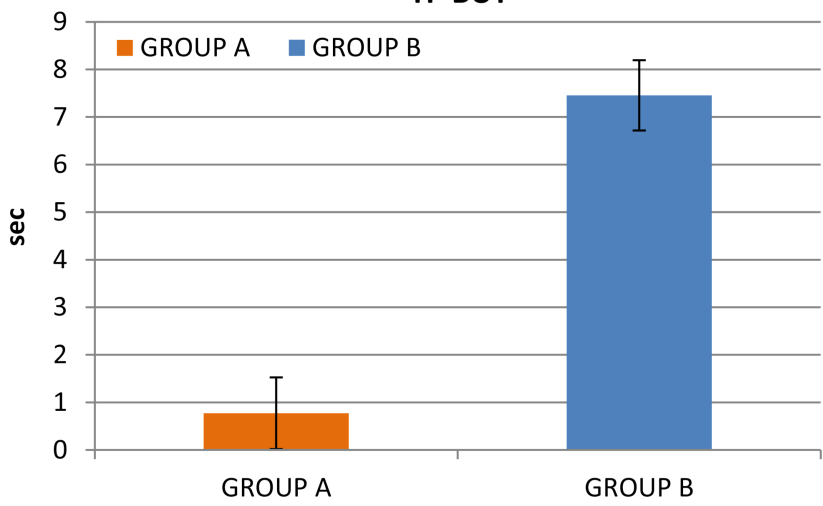

Figure 6 Statistically significant differences between the two groups for tear film break-up time (TF-BUT) values $(p<0.001 ; t=297419)$. Error bars represent SD.

tear production and restore the physiologic layer composition of tears.

A randomised clinical trial is needed to demonstrate the effectiveness of this approach.

Acknowledgements The authors are thankful to the following colleagues for working with them in this work: Drs Mario Toro, Santo Stella, Antonio Longo, Elina Ortisi, Silvia Caforio and Giuseppe Faro.

Collaborators Silvia Caforio, Elina Ortisi, Mario Toro, Antonio Longo, Santo Stella and Giuseppe Faro.

Contributors FD, TA and MR provided the critical review. CB and SC developed the manuscript. CG formulated the aims of the work, developed and revised the manuscript. GM and MVC elaborated data. RA, GN and GI collected the data and carried out the statistical analysis of the results.

Funding Financial support from Ministry of Education, University and Research (MIUR) (Grant N. 20078M2BFW/06).

\section{Competing interests None.}

Patient consent Obtained.

Ethics approval Azienda Ospedaliera Universitaria Vittorio Emanuele Policlinico Catania.

Provenance and peer review Not commissioned; externally peer reviewed.

\section{REFERENCES}

1 Knop E, Knop N, Millar T, et al. The International Workshop on Meibomian Gland Dysfunction. Report of the Subcommittee on Anatomy, Physiology, and Pathophysiology of the Meibomian Gland. Invest Ophthalmol Vis Sci 2011;52:1938-78.

2 Baudouin C. A new approach for better comprehension of diseases of the ocular surface. J Fr Ophthalmol 2007;30:239-46.

3 Tong L, Chaurasia SS, Mehta JS, et al. Screening for meibomian gland disease: its relation to dry eye subtypes and symptoms in a tertiary referral clinic in Singapore. Invest Ophthalmol Vis Sci 2010;51:3449-54.

4 Serrander A-M, Peek KE. Changes in contact lens comfort related to the menstrual cycle and menopause: a review of articles. J Am Optom Assoc 1993;64:162-6.

5 Verbeck B. Augenbefunde und stoffwech selverhalten bei einnahme von ovulation shemmern. Klin Monatsb/ Augenheilkd 1973;162:612-21.

6 Mathers WD, Stovall D, Lane JA, et al. Menopause and tear function: the influence of prolactine and sex hormones on human tear production. Cornea 1998;17:353-8.

7 Rocha EM, Wickham LA, da Silveira LA, et al. Identification of androgen receptor protein and $5 \alpha$-reductase mRNA in human ocular tissues. Br J Ophthalmol 2000;84:76-84.

8 Sullivan DA, Wickham LA, Krenzer KL, et al. Aqueous tear deficiency in Sjögren's syndrome: possible causes and potential treatment. In: Pleyer U, Hartmann C, Sterry W, eds. Oculodermal diseases-immunology of bullous oculo-mucocutaneous disorders. Buren, Netherlands: Aeolus Press, 1997:95-152.

9 Sullivan DA, Rocha EM, Ullman MD, et al. Androgen regulation of the meibomian gland. Adv Exp Med Biol 1998;438:327-31.

10 Saruya S. Studies on allergic conjunctivitis. Effects of castration and sex hormone administration on experimental allergic conjunctivitis. Acta Soc Ophthalmol Jap 1968:72:833-45 
11 Greenblatt RB, Colle ML, Mahesh VB. Ovarian and adrenal steroid production in the postmenopausal woman. Obstet Gynecol 1976;47:383-7.

12 Grodin JM, Siiteri PK, MacDonald PC. Source of estrogen production in postmenopausal women. J Clin Endocrinol Metab 1973;36:207-14.

13 Judd $H L$, Judd $G E$, Lucas WE, et al. Endocrine function of the postmenopausal ovary concentrations of androgens and estrogens in ovarian and peripheral vein blood. J Clin Endocrinol Metab 1974;39:1020-4.

14 Tsai TH, Scheving LE, Scheving LA, et al. Sex differences in circadian rhythms of several variables in lymphoreticular organs, liver, kidney, and corneal epithelium in adult CD2F1 mice. Anat Rec 1985;211:263-70.

15 Duarte MC, Pinto NC, Moreira $\mathrm{H}$, et al. Total testosterone level in postmenopausal women with dry eye. Arq Bras Oftalmol 2007;70:465-9.

16 Sullivan DA, Sullivan BD, Evans JE, et al. Androgen deficiency, meibomian gland dysfunction, and evaporative dry eye. Ann N Y Acad Sci 2002;966: 211-22.

17 Azzarolo AM, Wood RL, Mircheff AK, et al. Androgen influence on lacrimal gland apoptosis, necrosis, and lymphocytic infiltration. Invest Ophthalmol Vis Sci 1999:40:592-602.

18 Sullivan BD, Evans JE, Cermak JM, et al. Complete androgen insensitivity syndrome: effect on human meibomian gland secretions. Arch Ophthalmol 2002;120:1689-99.

19 Benelli U, Nardi M, Posarelli C, et al. Tear osmolarity measurement using TearLab Osmolarity System in the assessment of dry eye treatment effectiveness. Cont Lens Anterior Eye 2010;33:61-7.

20 Geerling G, Tauber J, Baudouin C, et al. The international workshop on meibomian gland dysfunction: report of the subcommittee on management and treatment of meibomain gland dysfunction. Invest Ophthalmol Vis Sci 2011;52:2050-64.

21 Shimazaki J, Sakata M, Tsubota K. Ocular surface changes and discomfort in patients with meibomian gland dysfunction. Arch Ophthalmol 1995;113:1266-70.
22 Tomlinson A, Bron AJ, Korb DR, et al. The international workshop on meibomian gland dysfunction: report of the diagnosis subcommittee. Invest Ophthalmol Vis Sci 2011;52:2006-49.

23 McCulley JP, Shine WE. Meibomian gland function and the tear lipid layer. Ocul Surf 2003:1:97-106

24 Foulks GN, Bron AJ. Meibomian gland dysfunction: a clinical scheme for description, diagnosis, classification, and grading. Ocul Surf 2003;1:107-26.

25 Suzuki T, Schirra F, Richards SM, et al. Estrogen and progesterone control of gene expression in the mouse meibomian gland. Invest Ophthalmol Vis Sci 2008:49:1797-808

26 Esmaeli B, Harvey JT, Hewlett B. Immunohistochemical evidence for estrogen receptors in meibomian glands. Ophthalmology 2000;107:180-4.

27 Yavas GF, Ozturk F, Kusbeci T, et al. Meibomian gland alterations in polycystic ovary syndrome. Curr Eye Res 2008;33:133-8.

28 Bonini S, Mantelli F, Moretti C, et al. Itchy-dry eye associated with polycystic ovary syndrome. Am J Ophthalmol 2007;143:763-71.

29 Kam WR, Sullivan DA. Neurotransmitter influence on human meibomian gland epithelial cells. Invest Ophthalmol Vis Sci 2011:52:8543-8.

30 Butovich IA, Millar TJ, Ham BM. Understanding and analyzing meibomian lipids-a review. Curr Eye Res 2008;33:405-20.

31 Suzuki T, Richards SM, Liu S, et al. Influence of sex on gene expression in human corneal epithelial cells. Mol Vis 2009;15:2554-69.

32 Biørnerem A, Straume B, Midtby M, et al. Endogenous sex hormones in relation to age, sex, lifestyle factors, and chronic diseases in a general population: the Tromsø Study. J Clin Endocrinol Metab 2004;89:6039-47.

33 Scuderi G, Contestabile MT, Gagliano C, et al. Effects of phytoestrogen supplementation in postmenopausal women with dry eye syndrome: a randomized clinical trial. Can J Ophthalmol 2012;47:489-92.

34 Avitabile T, Longo A, Caruso $\mathrm{S}$, et al. Changes in visual evoked potentials during the menstrual cycle in young women. Curr Eye Res 2007;32:999-1003. 